\title{
HORIZON SENSING FOR \\ ATTITUDE DETERMINATION
}

by Barbara K. Lande

\section{Introduction}

In space navigation, it is often necessary to determine the angles between the direction to the center of another body and the axes of the vehicle. The direction to the center of the other body may be found by calculating the direction which is perpendicular to the plane of its horizon. In order to determine this plane from a space vehicle, the angles between direction of the discontinuity between space and the body of interest and the axes of the vehicle are determined at several points or the horizon.

\section{Physicai Phenomena Which May Be Used to Define the Hoxizon}

There are several physical phenomena which make possible a variety of methods of sensing the discontinuity between space and the body of interest -- in other words, the horizon.

\section{A. Infrared Radiation from the Earth}

If earth is the body of interest, one physical phenomenon which differentiates it from space is the infrared radiation it emits. The earth's horizon may be defined as the sharp gradient of infrared $r$ adiation which exists at the limb, or border, between it and outer space.

Since the earth has a fairly uniform temperature this gradient may be used for space navigation under a wide variety of circumstances, whether or not the limb is illuminated by the sun. In other words, it may be used under both day and night conditions. Figure 1 shows the output from an infrared sensor which was mounted in TIROS III, a weather satellite. As the satellite rotated, the field of view of the sensor scanned first over the sky, then over the earth, then over the sky again. As the sensor's field of view passed over the horizon, it detected the shaxp change in the level of infrared radiation which is represented by the sharp rise in the signal. As it passed over the opposite horizon of the earth, it detected the sharp decrease in the level of infrared radiation. It should be emphasized that this figure represents fairly ideal conditions. This sharp gradient of infrared radiation, which exists at the boundary between earth and space 
beyond, is the physical phenomenon most commoniy used at present for sensing the horizon in space navigation.

\section{B. Albedo from the Earth}

The sunlight which is reflected by a body in space such as the earth, sometimes referred to as the aibedo, is another phenomenon which has been used for horizon determination. However, it is limited in application. At night, when the earth is eclipsing the sun with respect to a space vehicle, no reflected sunlight appears. At other times, the earth appear 8 to have phases like those of the moon, complicating the calculation of the horizon plane by means of the albedo which, incidentally, is defined as the ratio between the light received and the light reflected by a body in space. Figure 2, a colored photograph taken during one of the Mercury sub-orbital flights, illustrates this phenomenon. The horizon is easily seen as the gradient between the apparent surface of the earth which reflects sunlight, and black space beyond, which does not. The Georgia-Florida coast line is also visible in the picture.

\section{Air Glow Around the Earth}

The upper portion of the earth's atmosphere radiates due to excitation by the sun. This phenomenon, known as air glow, could be used to define the horizon. The radiation occurs both day and night.

The total visible intensity of this radiation of air glow is approximately equal to the total amount of starlight aeen on a clear but moonless night. Air glow is rarely observed on the surface of the earth because it is evenly distributed throughout the sky. Much of the illumination by air glow is in a few spectral lines. Figure 3 shows soms experimental data on the air glow in the sodium line at $5893 \AA$. This is one of the more intense and better known lines. Its intensity during the day is about seventy -five times the nighttime intensity. This air glow radiation comes from a rather narrow band in the atmosphere occurring approximately 90 kilometers above the surface in a layer of about 10 kilometer 8 in thickness. Sor. \& scientists think that if the earth were viewed from a long distance away, with a sensor which is sensitive to only one particular band of air glow, a doughnut of radiation would be seen circling a dark earth while the rest of space would be dark, except for point sources. The earth's horizon then could be determined by measuring the physical position of the air glow band. Very few measurements have been made utilizing the air glow phenomenon. 
Some of the most recent data indicate air glow's intensity varies from point to point over the earth's surface. Its altitude also appears to vary with time. Altitude measurements have been taken only at White Sands, New Mexico. Looking at Figure 2 again, the picture taken during the sub-orbital Mercury flight, we can see no evidence of the aix glow phenomenon. The intensity of aix glow in the vitra violet is only approximately one ten-billionth of the thermal energy the earth radiates. This illustrates one of the main limitations on the usefulness of air glow for space navigational purposes.

\section{Other Bodies}

The horizons of bodies other than the earth may be defined by use of the same general phenorriena already discussed, namely:

1. emitted thermal radiation;

2. reflected radiation (albedo); or

3. other phenomena such as air glow.

The horizons of the nearby planets, Venus and Mars, and perhaps artificial satellites, may be determined by sensing the gradient between the infrared radiation which they emit and that of space.

The visible radiation emitted by the photosphere of the sun may be used to determine its horizon. The horizons of the moon, the sear planets, and perhaps artificial satellites also may be determined by the gradient of reflected sunlight at certain times.

\section{Scanning Schemes}

There are 5 scanning schemes which have been used or proposed for determining the direction of the local vertical.

\section{A. Passive Scan}

The passive scan can only be used on a space vehicle which rotates. A sensor with a small field of view is mounted in vehicle with its field of view perpendicular to the spin axis of the vehicle. As the vehicle rotates, the field of view of the sensor is swept through space. 
The data in Figure 1 were taken with a scanning scheme similar to this. The times at which the field of view of the scanner pase the two horizons can be determined with the use of this data. From knowledge of these time parameters the orbit of the vehicle at any instant can be determined. This technique was employed using infrared sensors on the TIROS weather satellite, and will be used on the Godrard Space Flight Center atmospheric structure S-6 satellite. It has also been employed on daytime rocket shots using sensors which detect the sur's reflected light.

\section{B. Conical Scan}

A second type of scanning method is the conical scan. Figure 4 shows the Mercury space capsule. The cones represent the fields of view which its horizon scanner sensors sweep out in space. The Mercury capsule has two scanners which are used to determine its pitch and roll errors. Each scanner, again, has a small field of view. This time the rotation of the field of view is done in the scanner itself. The field of view is rotated in a circle, it scans a cone out of the space. This cone inter sects the earth and the ontput of the sensor is used to determine the location of the gradient of infrared radiation between space and the earth. The local vertical can then be computed. It is presently planned that the type of scan shown here for the Mercury vehicle will be used in the Nimbus advanced weather satellite. The scan pattern is not very efficient from an information theory point of view. The information as to the direction of the horizon is only a very small part of the information which enters the sensor.

\section{Linear Scan}

Figure 5 shows a third type of scanning pattern, the linear scan. This; slide shows the Orbiting Geophysical Observatory satellite and the pattern the fields of view of its horizon scanner sensors sweep out in space. This satellite employs the linear type scan. In this case, there are 4 sensors on the vehicle and they each have small fields of view. These fields of view are rotated in a plane from space until they intersect the edge of the earth. They are oscillated at the edge of the earth for the rest of the flight time. The angle between the direction of the fields of view of each sensor, once they have locked on the edge of the earth, and the satellite can be determined and the direction of the local vertical can be calculated. This type of scanning is better from an information theory point of view than the circular type scan because the sensor field of view spends more of its time right at the edge of the 


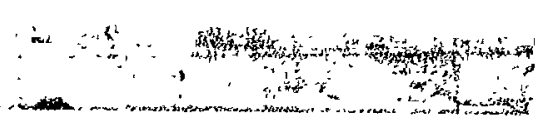

earth. Only infrared sensors have been used with t: ese two methods of scanning.

\section{Nutating Scan}

A fourth type of scanning is called nutating scan. It is sirnilar to a linear scan but there is only one sensor per vehicle. The field of view of the sensor is again small and is initially viewing space. It is swept down until it intersects the horizon. It then oscillates about the hori207. Meanwhile the whole scanning head is rotated about another axis. Thus the projection of the sensor field of view on the earth is a sinusoidal pattern along the earth's horizon. The direction of the local vertical is then determined by calculations using the position of the field of view of the sensor with respect to the vehicle at each instant.

\section{E. Illumination Comparison}

A fifth type of horizon sensing does not use any moving parts. The illumination from one side of the earth on the scanner is compared with that from the other side of the earth. This information is used to deter mine the local vertical. For a vehicle within 1000 miles of the earth, it is very difficult to image the whole earth on the scanner because such an imaging system would have to have a very wide field of view. A scanner which uses this method has been preposed. It uses an infrared sensor and a unique imaging system. Another scanner which employs this method was used on the Ranger Moon vehicle. It determined the direction to the earth from distances greater than 80,000 miles. It used sensors sensitive to visible light.

\section{Programs Which Use Horizon Scanning}

Programs which have used horizon sensors for attitude determination are spinning satellites and rockets including the Goddard S-6 atmospheric structure satellite and the TIROS satellite. The Mercury man in space program has used horizon scanners. Horizon scanners have been used to monitor the performance of some rockets. They are also used in the Air Force Agena rocket and satellite system. The Jet Propulsion Laboratory has used an earth sensor in its Ranger vehicle.

Future programs which have planned to use earth horizon scanners are the Orbiting Geophysical Observatory, the Nimbus advanced weather satellite, the Gemmini manned vehicle, the Apollo manned 


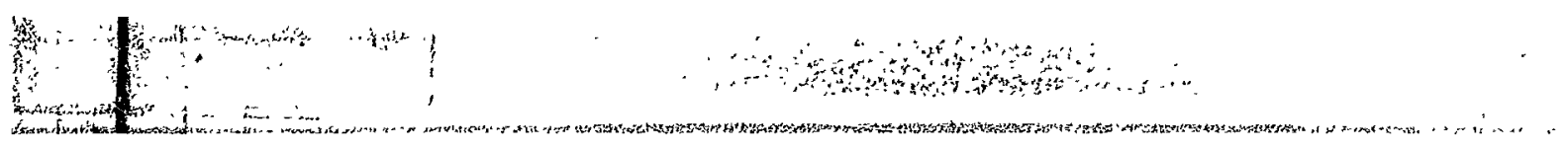

vehicle, classified programs for the Air Force and the Saturn rowkets testing program.

Future programs for which the earth horizon scanners are under consideration are the Communication Satellites and short lived rocket probes. Another future use for horizon scanners is on the Mariner vehicles for trips to Venus and Mars. Horizon scanness would determine the direction between local vertical of those planets and the vehicle axes. Another use for horizon scanners is tor the Advanced Orbiting Solar Observatory. This satellite is planned to orbit the earth but observe the sun. An earth horizon scanner could be used to cetermine the direction to che center of the earth. A sun horizon scanner or limb sensor could be used to determine precisely the direction to the center of the solar disk. A moon horizon scanner may be used on lurax mis sions such as Apollo.

\section{Horizon Scanning Accuracy}

There are two basic limitations to the accuracy of any deternination. One source of inaccuracy is errors developed in the instrument which makes the determination and the other source of error is variability in the physical phenomenon which is utilized for the determination.

\section{A. Instrument Error}

In the case of horizon scainers, the sources of instrument error are detector noise, mechanical tolerances, and mounting rigidity. The detector noise is more serious when using some types of scanning patterns than others. Detection noise can be made to have as small a contribution to the error of the attitude determination as desired by using larger oftics, more sophisticated scanaing methods and better detectors. Mechanical tolerances in scanners can be made better than a few seconds of arc, if necessary, for good mechanical designs. In other words, the exror in determining the local vertical can be made smaller than a few seconds of arc due to mechanical tolerances of the scanner. Mounting rigidity can be a problem in some space vehicles. Errors can be developed as high as $1^{\circ}$ if care is not taken. However, if it is deaired to align an experiment with a heavenly body, alignment can be as good as $1 / 10$ of a second of arc if the radiation coming into the experiment is shared with the attitude sensow; 


\section{B. Variability in Physical Phenomena}

The other class of phenomena which cause errors in detecting locai vertical are those due to the heaveniy body in question. Errors which are dependent on the physice of the body generally are a certain magnitude of the apparent diameter of the body and decrease in absolute magnitude as the scanner gets farther away from the body because of the apparent decrease in absolute angular diameter. The maximum error due to the ellipticity or oblateness of the surface of the earth in determining the direction of the horizon at one point is nct greater than 1/10 of a degree. This error could be made zero if one could take the ellipticity of the earth into account when calculating the attitude. Much larger errors can arise due to irregularities in the apparent surface of the earth, such as might be caused by high clouds. These may be as large as 1/4 of a degree at an altitude of $200 \mathrm{n.mi}$. but may be mitigated somewhat by the refraction by the atmosphere of the radiation from tho tops of the clouds.

Another source of exror for some types of scan is cold clouds appearing on the face of the earth. Figure 6 shows recordings from sensors in the horizon scanner 3 in one of the Mercury flights, MA-5. In the second scan illustrated, the field of view of the sensor passed over a very cold cloud on the face of the earth. The temperature of the cloud appeared to be about $225^{\circ} \mathrm{K}$. Clouds coldex than $200^{\circ} \mathrm{K}$ were observed. This type of cloud emits only about $1 / 2$ of the total $\mathrm{x}$ adiation emitted by an average area of the earth and only $1 / 4$ ilof the radiation with wavelengths between 8 and 12 microns as. a.verage area of the earth radiates. These clouds could cause a great error in the attitude determined by any scanner whose field of view crossed thern. The scanner might con'se the cloud with the horizon of the earth. There are scanning $r$,nods which are not bothered by this type of cloud appearing on the face of the earth. They would be bothered by such a cloud appearing at the horizon but the cloud would have a amaller apparent diameter and its radiation would be augmented by the atmosphere through which it would be viewed.

Dr. Rudolph Hanel of the Goddard Space Flight Center, Meteorology Division, has suggested that by using a scanner sensitive only to wavelengths around 15 microns, which are strongly absorbed by carbon dioxide, the phenomena of cold clouds could be avoided. Radiations in these frequencies should come from very high in the atmoaphere at the top of the carbon dioxide region, above the level of cold clouds. 
The trace on the right in Figure 6 was taken at a different time, when the sensor field of view passed near the sur. The signal from the sun appears to the right on the picture. The amplitude of the signal from the sur is clearly stronger from that of the earth, but the scanner could think that the onset of the signal from the sun was the horizon of the earth and an attitude determination from this data could be in error by many degrees. It is interesting to note from this data that the apparent diameter of the sun is about $73^{\circ}$ which is much larger, of course, than the apparent diameter of the visible sun in the sky which is $1 / 2$ degree.

TIROS, the weather satellite, carried a 5 channel radiometer which sensed radiations from the earth in 5 different spectral bands. The next slide shows an example of the signals from the 5 channel radiometer. The first signal on this slide is from the radiometer which was sensitive to radiations with $61 / 2$ micron wavelength. T'his type of radiation is strongly absorbed by water vapor so the signal in this channel is from the top of the water vapor in the atmospinere. The signal from this channel shows severe limb darkening. In other words, the waves shape from the sensor as it scans over the earth appears sometimes almost triangular $r$ ather than square and there is a smaller gradient of radiation at the horizon. The second line shows the signal from the radiometer which is sensitive to radiation of wavelengths $c f$ 8 to 12 microns. This is considered to be the water vapor viridow and the radiation recorded here probably came from near the surface or from extremely opaque clouds. The first slide was an enlargement of the signal from this channel. Channels 3 and 5 were chiefly sensitive to visible light and were used as a check on the TV camera in the satellite. The radiometer whose output here is channel. 4 was sensitive to radiation wavelengths from 8 to 30 microns. The signal in this channel $i_{\omega}$ very similar to that of channel 2 whose radiometer was sensitive to 8 to 12 rnicron radiation. The next slide show 8 another enlarged.view of a signal from channel 2. The signals frum the TIROS 5 channel radiometer show the cold clouds and sharp gradients on the surface of the earth which also appear in the signals from Mercury horizon scanner shown earlier.

The angular width of the signal from the earth was measured for 34 consecutive scans of the channel 2 radiometer. A level of about one-fifth of the maxinum signal level was chosen as the "slicing level." The diameter of the earuh appeared to vary from scan to scan. The rms variation was 1.8 angular degrees. This variation can be completely attributed to system noise. This variation in apparent diameter 
would cause er rors of this approximate magnitude in attitudes detexmined from this data.

Mars is a planet which would look much like the sarts to an infrared horizon scanner. It probably has few water clouds but it may have high, opaque dust clouds. In fact, one interesting, and as yet unexplained, fact about $\mathrm{Mar} ;$ is that its ellipticity, or oblateness, as calculated from the orbits of its planets is one half that which $c$ a be measured on photographs of the planet. A reasonable hypothesis which explains this anomaly is that the solid surface conforms to the ellipticity predicted by the motion of the satellites, and the apparent bulge around the equator is caused by dust clouds which appear there. The actual ellipticity of Mars is somewhat less than that of the earth, but the apparent ellipticity of Mars is almost twice that of the earth.

This, or some other similar phenomena could also occur on earth causing errors in horizon scanners. The apparent shape of the earth when viewed with radiation of certain wavelengths and the consistency of that shape with time has not been determined experimentally for the earth.

In the past the attitude of spacecraft with recpect to the sun has been determined by sensors which use the illumination from the total solar disk, However, sun spots can have an area equal to 1/300 of the total disk of the sun. A zensor which compares radiation from the two halves of the solar disk could be thrown off by 5 seconds of arc. For a really precise determination of the direction of the sun, some sort of limb tracking or horizon sensing of the sun must be used.

\section{Experimentally Determined Accuracy}

The TIROS satellite also carried another passive scan infrared sensitive horizon scanner. The attitude of the satellite was calculated by reducing the horizon scanner data and also from the horizons and other land marks appearing on the TV pictures from the satellite.

By hand selecting the best data from the scanner and smoothing it with a computer, the attitude determined using the horizon scanner data agrees with that determined using the TV pictures to one or two degrees. The attitude determined using the TV pictures is considered to be more accurate. 


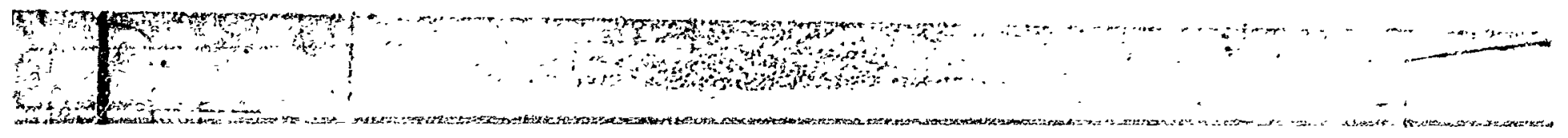

The horizon scannex on the Mercury capsule was specified to be accr sate to one degree of arc. The recorded signals from its sensor show that it did not meet this requirement at cextain times.

\section{Conclusion}

In conciusion, horizon scanners for attitude determination, up to now, have been limited by instrument accuracy rather than by errors due to the body which they were scanning. The accuracy of scanners. which have been flown have been at bast one or two degrees. It appears that accuracy of $2 / 10$ of a degree for altitudes below 1000 miles and $1 / 10$ of a Jegree for altitudes above 1000 miles are attainable using techniques which are now in development.

The use of physical phenomena other than the total infrared radiation from the earth, such as aix glow or narrow spectral band infrared radiation, may be necessary to attain better accuracy. More information on these phenomena needs to be known before horizon scanners can be designed to use them. 


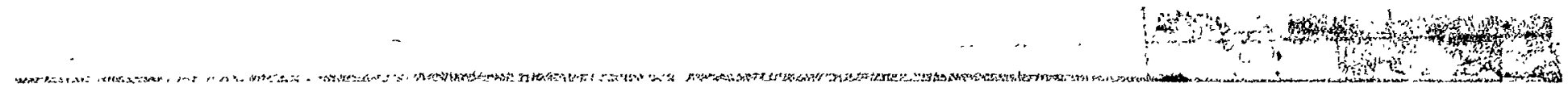

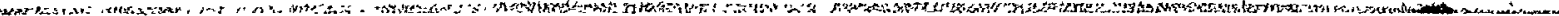

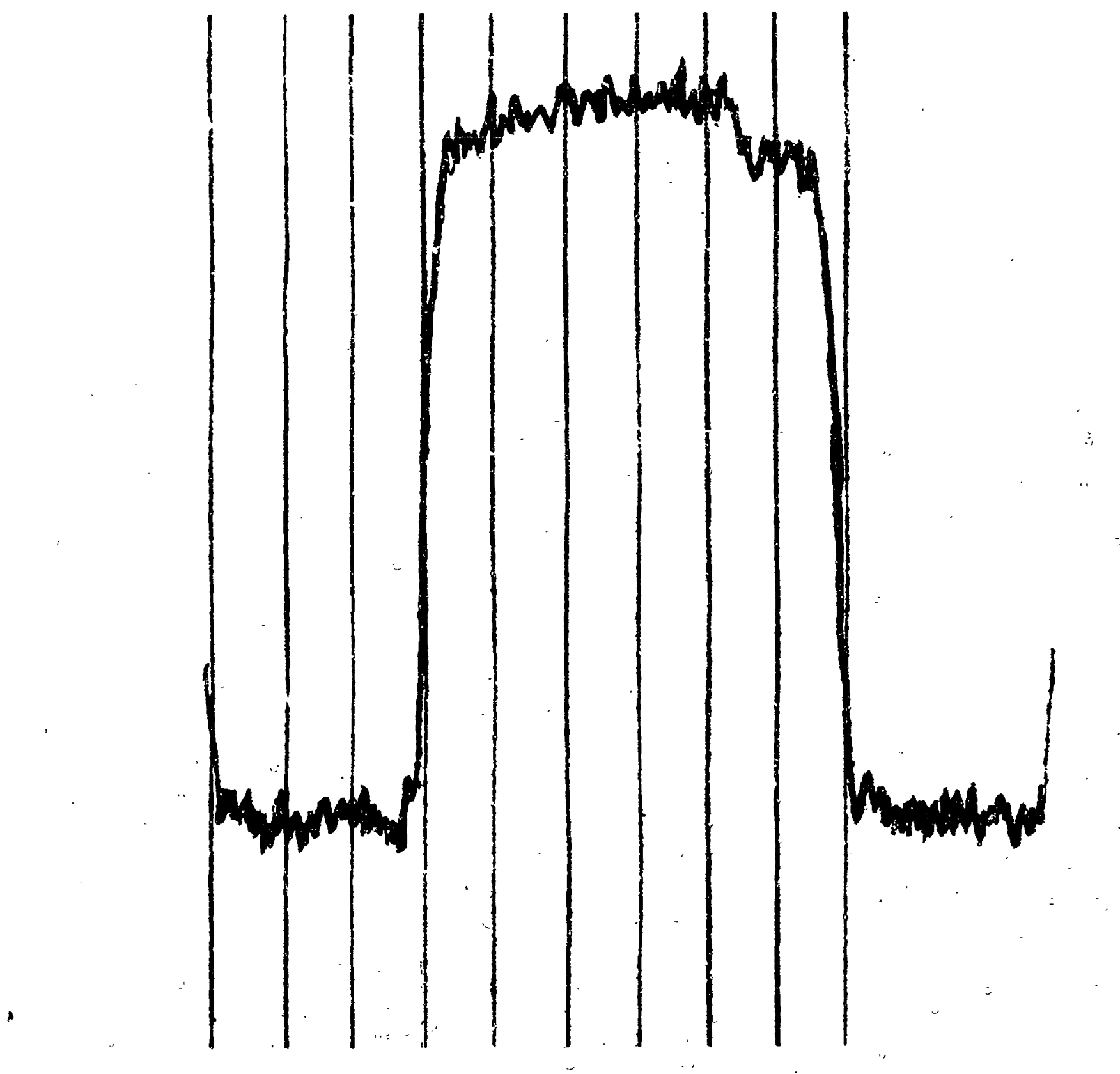


1

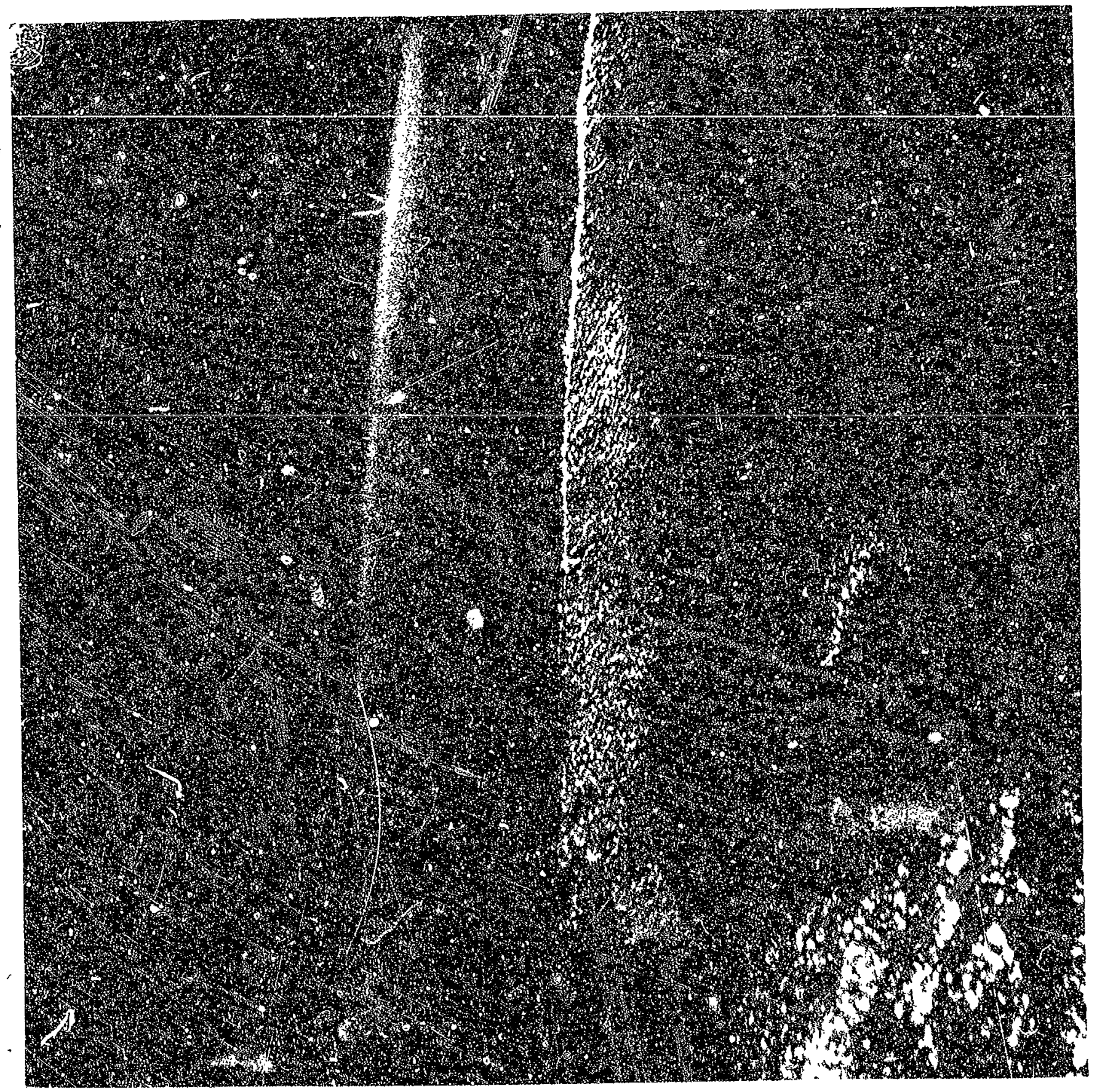




\section{AIR GLOW}

Intensity and Altitude of the Sodium 5893 A Line

\section{INTENSITY \\ (relative magnitude)}

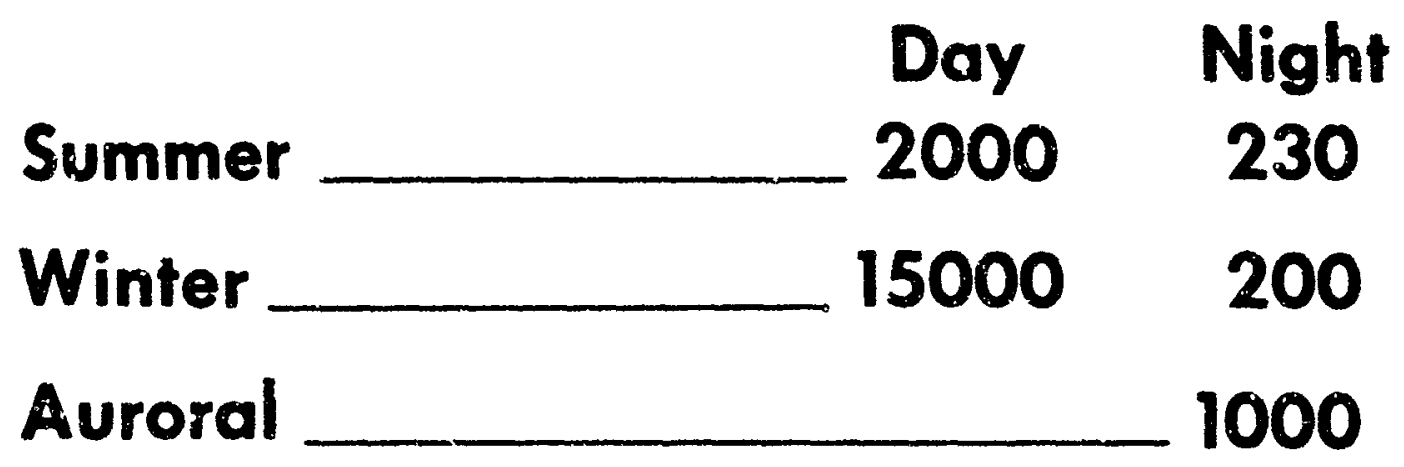

\section{ALTITUDE}

(two measurements at White Sands, N.M.)

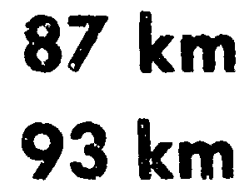


1

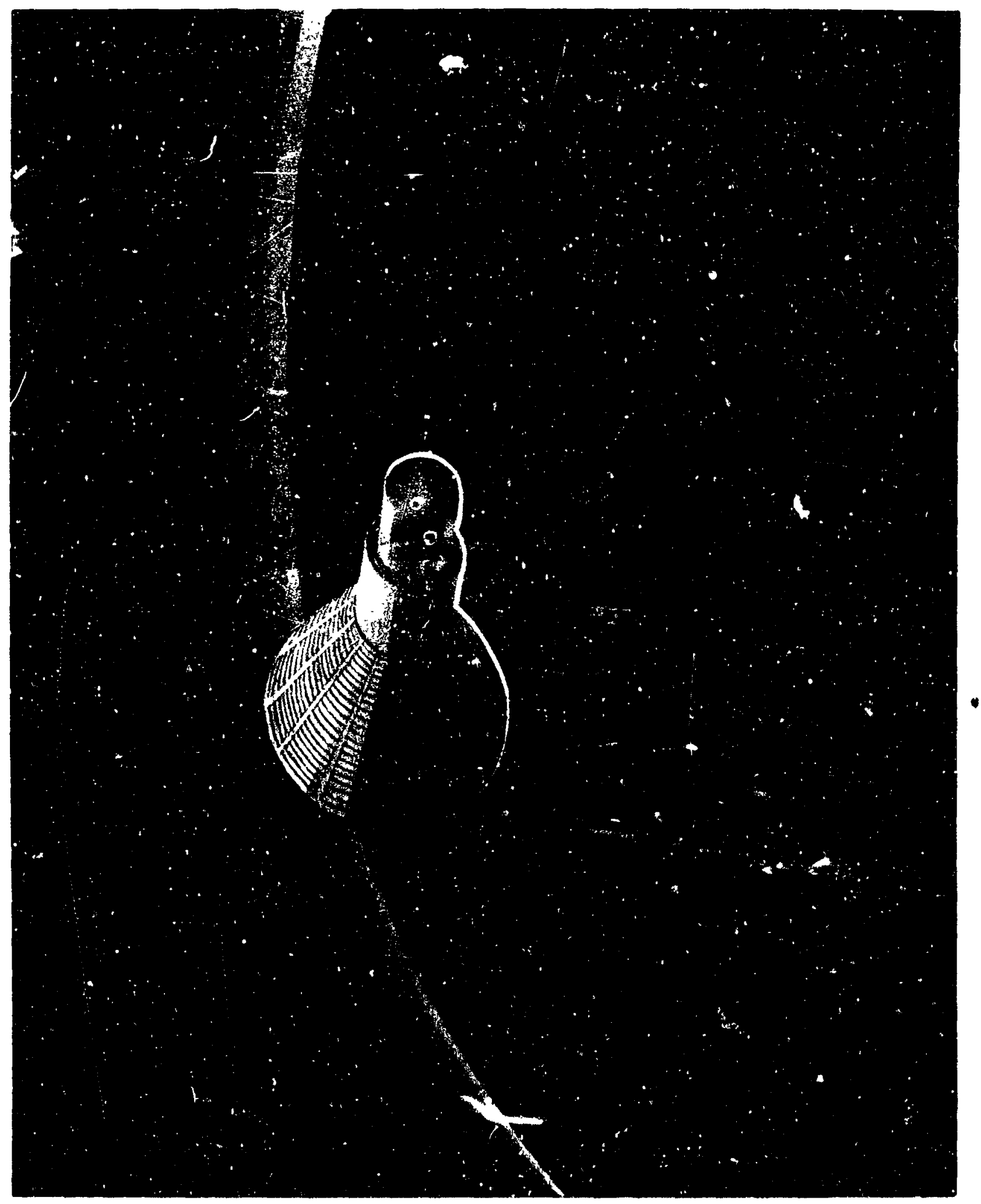




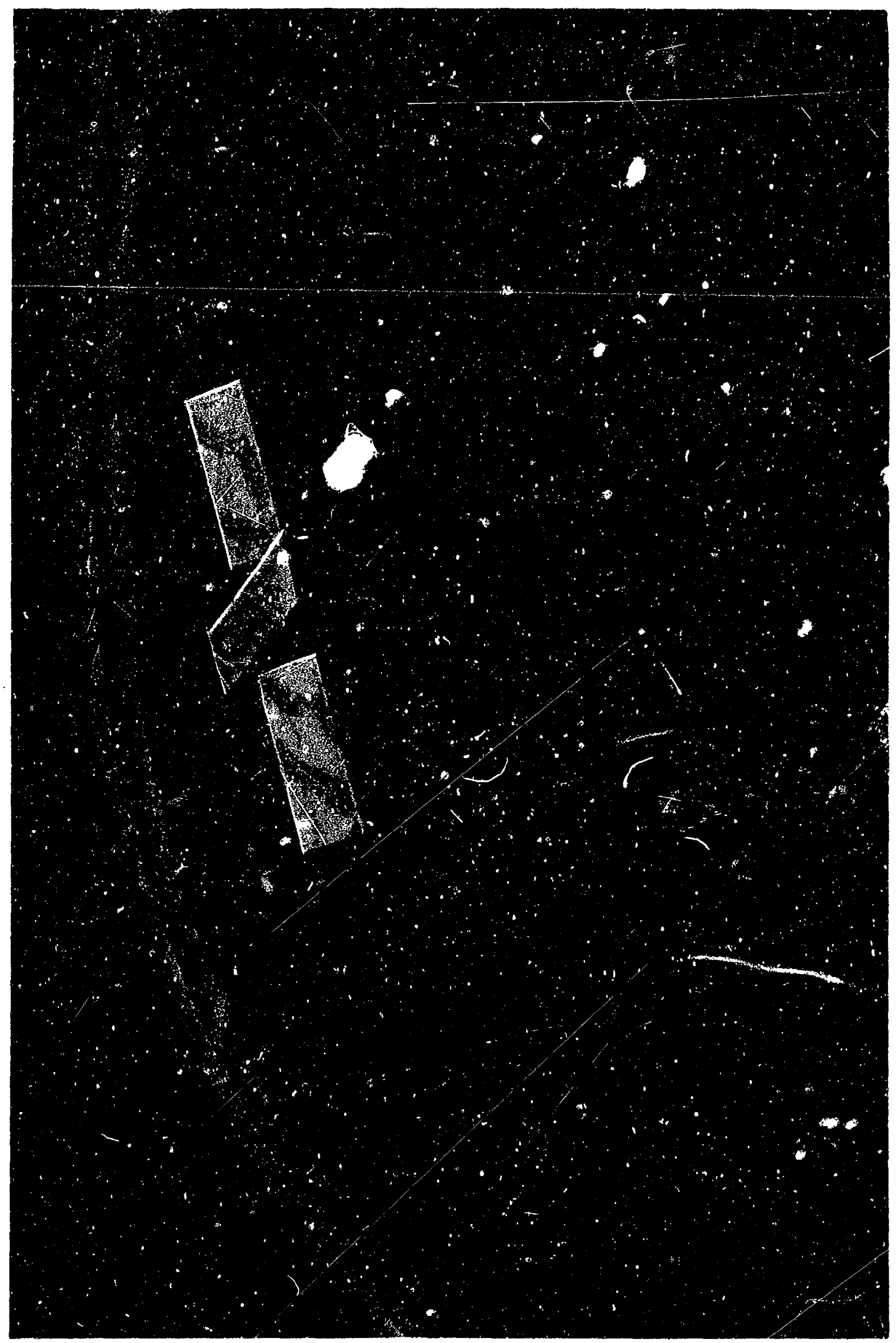




\section{SAMPLES OF HORIZON SENSOR SIGNALS FROM MERCURY SCANNER}
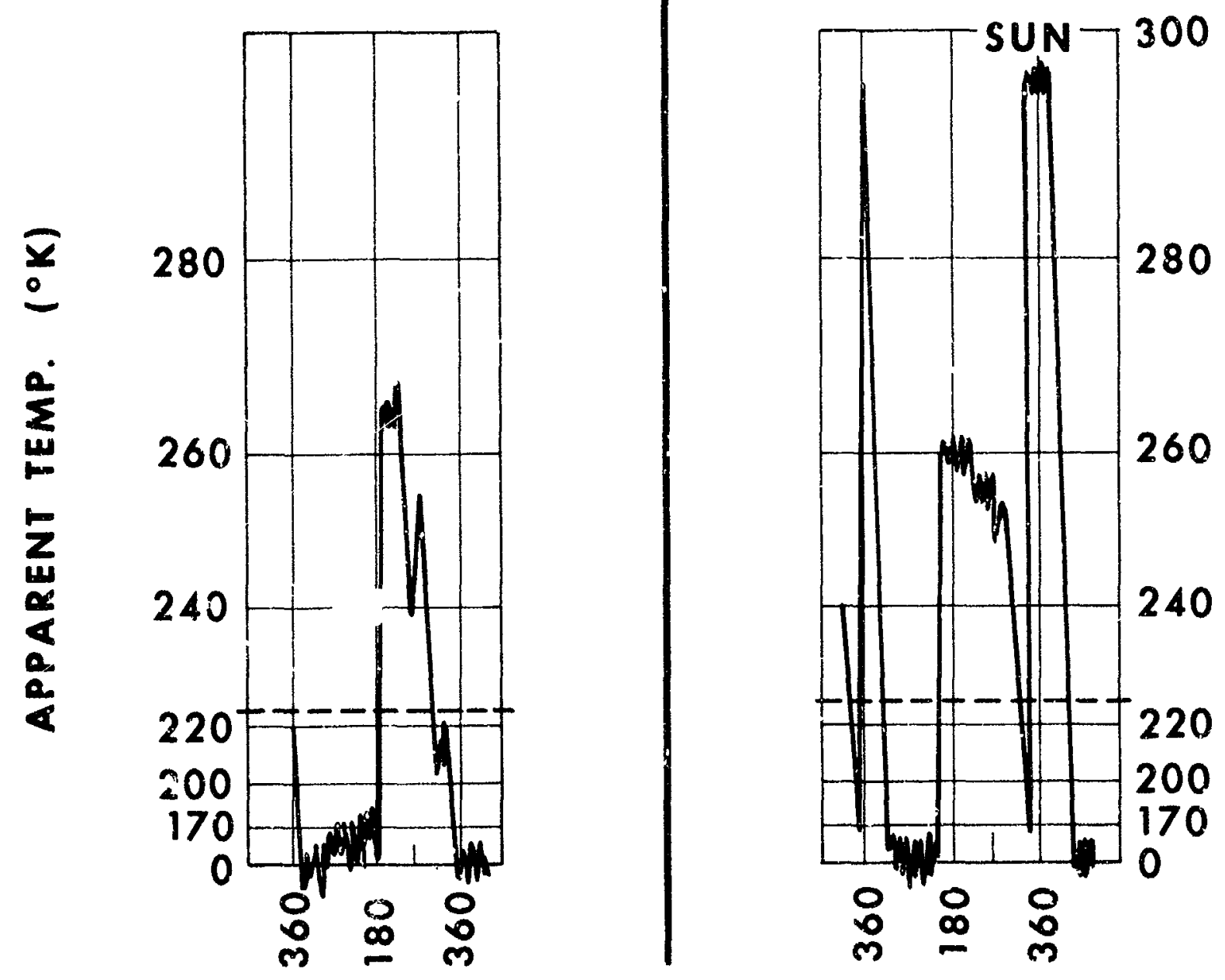

DEGREES OF SCAN 


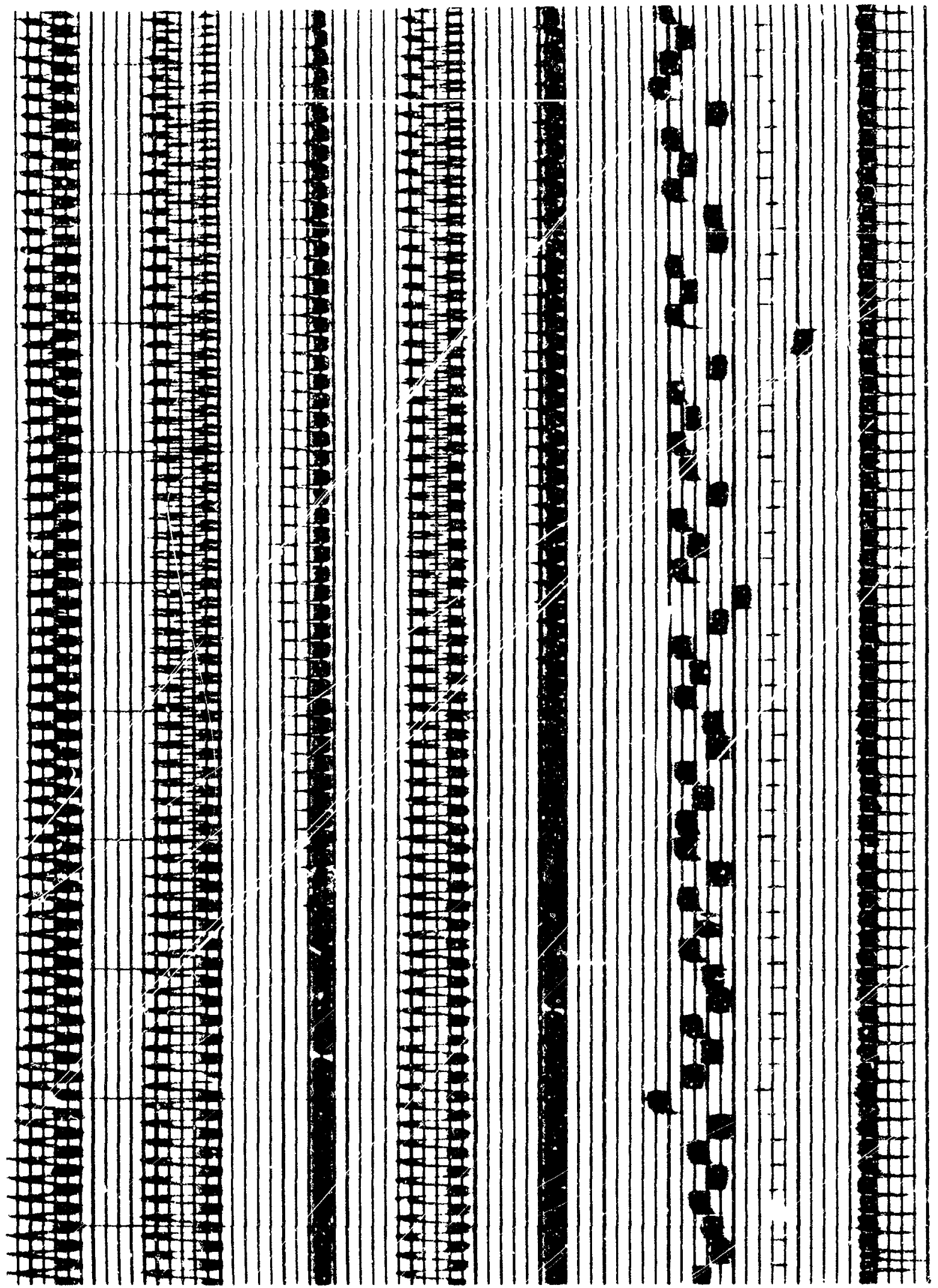


1

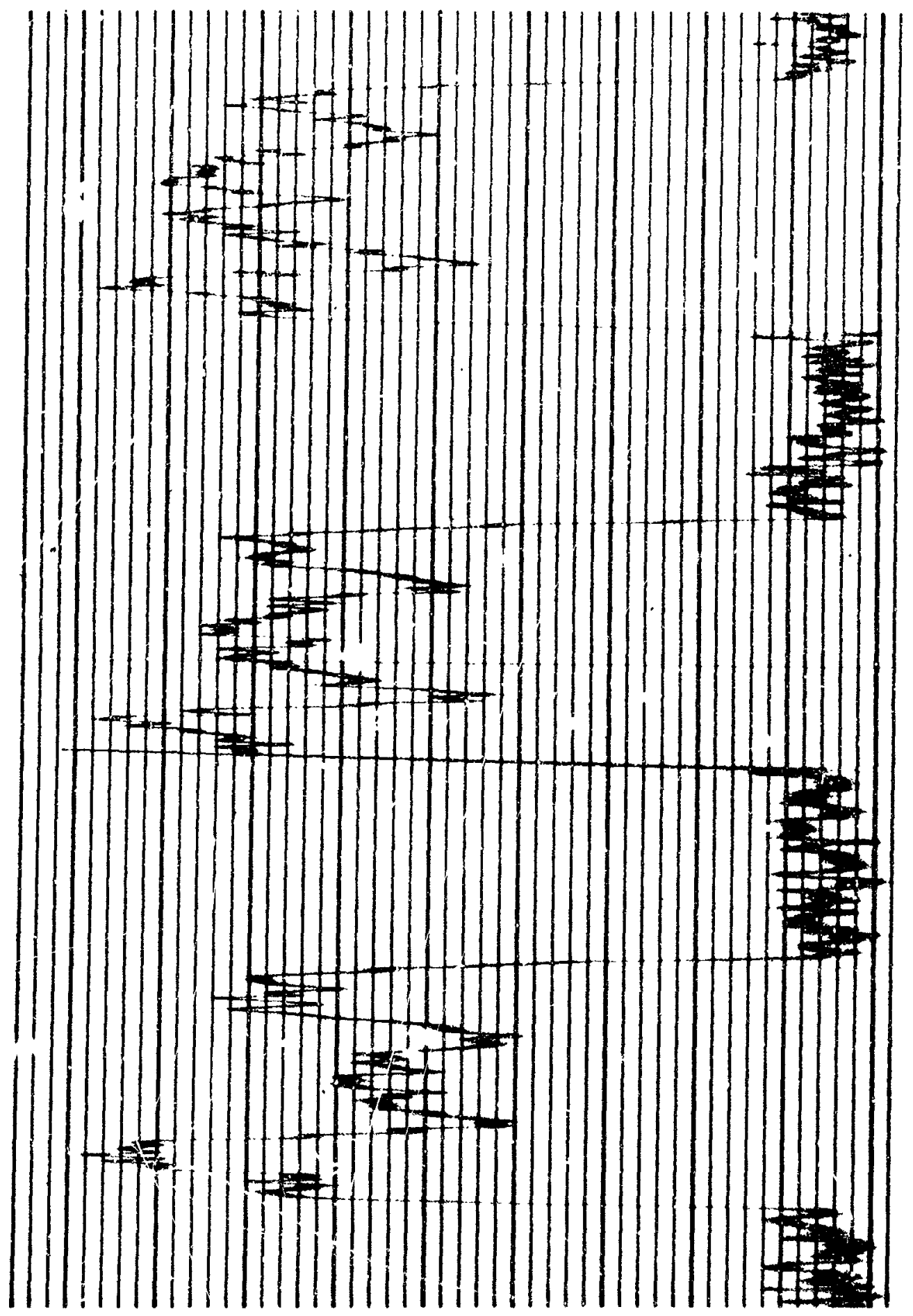

